Revue musicale OICRM

revue musicale oicrm

\title{
Les écrits de compositeurs dans les publications liées au Congrès pour la liberté de la culture (1952-1966)
}

\section{Anne-Sylvie Barthel-Calvet}

Volume 7, numéro 1, 2020

URI : https://id.erudit.org/iderudit/1069479ar

DOI : https://doi.org/10.7202/1069479ar

Aller au sommaire du numéro

\section{Éditeur(s)}

Observatoire interdisciplinaire de création et recherche en musique (OICRM)

\section{ISSN}

2368-7061 (numérique)

Découvrir la revue

\section{Citer cet article}

Barthel-Calvet, A.-S. (2020). Les écrits de compositeurs dans les publications liées au Congrès pour la liberté de la culture (1952-1966). Revue musicale OICRM, 7(1), 179-189. https://doi.org/10.7202/1069479ar
Résumé de l'article

Dans le contexte culturel de la guerre froide, le Congrès pour la liberté de la culture met en place une politique médiatique offensive, en particulier à travers un bouquet de journaux implantés dans divers pays européens, dont Preuves en France. D'autres publications, comme La Revue Musicale sont sollicitées pour des numéros spéciaux. C'est ainsi qu'en 1952, à l'occasion du festival "L'Oeuvre du Xx ${ }^{\mathrm{e}}$ siècle ", et en 1965-1966, avec l'enquête " La musique sérielle aujourd'hui » menée par André Boucourechliev, des textes de compositeurs sont rassemblés, respectivement dans La Revue Musicale et Preuves. Les auteurs sont invités plus ou moins explicitement à aborder des questions comme l'évolution en cours de la musique. D’une grande diversité de ton comme de contenu, ces écrits traduisent des conceptions très différentes du rapport au grand public. Cet article se propose d'étudier la manière dont les compositeurs ont investi ces espaces médiatiques spécifiques. 


\title{
Les écrits de compositeurs dans les publications liées au Congrès pour la liberté de la culture (1952-1966)
}

\author{
Anne-Sylvie Barthel-Calvet
}

\begin{abstract}
Résumé
Dans le contexte culturel de la guerre froide, le Congrès pour la liberté de la culture met en place une politique médiatique offensive, en particulier à travers un bouquet de journaux implantés dans divers pays européens, dont Preuves en France. D'autres publications, comme La Revue Musicale sont sollicitées pour des numéros spéciaux. C'est ainsi qu'en 1952, à l'occasion du festival « L'Euvre du Xx ${ }^{e}$ siècle ", et en 1965-1966, avec l'enquête "La musique sérielle aujourd'hui " menée par André Boucourechliev, des textes de compositeurs sont rassemblés, respectivement dans La Revue Musicale et Preuves. Les auteurs sont invités plus ou moins explicitement à aborder des questions comme l'évolution en cours de la musique. D'une grande diversité de ton comme de contenu, ces écrits traduisent des conceptions très différentes du rapport au grand public. Cet article se propose d'étudier la manière dont les compositeurs ont investi ces espaces médiatiques spécifiques.
\end{abstract}

Mots clés : guerre froide ; musique contemporaine ; politique culturelle ; sérialisme ; $\mathrm{Xx}^{\mathrm{e}}$ siècle.

\begin{abstract}
In the cultural Cold War context of competition between Communist-aligned World Peace Council and American-supported Congress for Cultural Freedom, the latter developed an offensive media policy, in particular with a bunch of specific journals in some European country, among others Preuves in France. Links were also built with other reviews for special issues, like La Revue Musicale. Twice, in 1952, on the occasion of the festival "L'Euvre du Xx' siècle," and in 1965-1966, with the survey "La musique sérielle aujourd'hui" ("Serial music today") conducted by André Boucourechliev, composers' texts about musical creation were gathered, respectively in La Revue Musicale and Preuves. Composers were asked - more or less explicitly— to express their opinion on the current evolution of music, generally or under a specific problematic. These writings display a great diversity in tone and content and reflect various ways to address the general public. This paper considers how composers enter this media field.
\end{abstract}

Keywords: Cold War; cultural politics; New Music; serialism; 20 $0^{\text {th }}$ century. 
Dans le contexte de radicalisation de la guerre froide et d'implication politique des intellectuels européens, deux organisations tentent de rivaliser d'influences : d'une part, le Conseil mondial de la paix, d'obédience communiste, dont la première manifestation fut le Congrès mondial des intellectuels pour la paix de Wrocław, et d'autre part, le Congrès pour la liberté de la culture. Sur le plan musical, une émanation de la première tendance est le second Congrès international des compositeurs et des critiques musicaux qui se tient à Prague en mai 1948 et qui rédige ce que l'on a appelé le Manifeste de Prague définissant les principes d'un "réalisme socialiste musical "; Serge Nigg et Louis Saguer, du groupe des " Musiciens progressistes » (avec Roger Désormière, Louis Durey et Elsa Baraine) sont d'ailleurs signataires de ce Manifeste. Du côté anticommuniste, le Congrès pour la liberté de la culture impose sa première marque par une action indéniablement spectaculaire : le festival " L'Euvre du $\mathrm{XX}^{\mathrm{e}}$ siècle " organisé par le compositeur américain d'origine russe Nicolas Nabokov, nommé Secrétaire général de l'organisation sur la proposition de ce projet (Grémion 1995, p. 72-73 ; Giroud 2015, p. 251-252). Ce festival impliquant différentes manifestations intellectuelles et artistiques (concerts, exposition d'art moderne, débats intellectuels et littéraires) se déroule en avril-mai 1952 à Paris et vise à obtenir, par la collaboration d'institutions américaines et européennes du plus haut niveau, un impact décisif dans le Kulturkampf contre l'URss (Carroll 2003, p. 69-70). La programmation musicale est d'un faste inouï puisqu'elle rassemble en un mois des institutions aussi prestigieuses que l'Orchestre symphonique de Boston, l'Opéra de Vienne, l'Orchestre du Théâtre national de l'Opéra de Paris, l'Opéra royal de Covent Garden ou le New York City Ballet, et des chefs d'orchestre de renommée internationale (Munch, Böhm, Stravinski, Britten, Walter, Monteux, Ansermet, Fricsay). Le programme symphonique et lyrique vise à faire découvrir au public parisien des chefs-d'œuvre de la première moitié du siècle tels que Wozzeck, Erwartung ou le Chant de la Terre ; le Sacre du printemps est redonné dans le lieu de sa scandaleuse création et sous la baguette du même Monteux (ibid., p. 177-183). Le programme de musique de chambre est davantage tourné vers la création contemporaine avec, entre autres, les Structures Ia pour deux pianos de Boulez, les Visions de l'Amen de Messiaen ou Choral et variations pour piano de Dutilleux (ibid., p. 183-185).

Par la suite, le Congrès pour la liberté de la culture manifeste son soutien à la vie artistique et littéraire internationale avec un système de bourses pour les jeunes ou en favorisant des rencontres internationales telles que le East-West Music Encounter à Tokyo en 1961 où se retrouvent des compositeurs tels que Carter, Xenakis, Berio, Cowell ou encore Takemitsu.

Son combat culturel et idéologique passe également par le développement d'organes de presse dans les différents pays où il mène son action : Der Monat, d'abord en Allemagne à partir de 1948, puis Preuves, né en France en 1951, The Encounter au Royaume-Uni ou encore Tempo en Italie (Grémion 1995, p. 145-148). Ces revues généralistes d'actualité abordent des questions sociales, politiques, économiques et culturelles, qui s'adressent à un public cultivé. Leur affiliation est clairement affichée, comme il est annoncé sur la page de garde de Preuves : "revue mensuelle publiée sous les auspices du Congrès pour la liberté de la culture ». La parole y est régulièrement donnée à des acteurs de premier plan des domaines concernés. Pour la 
musique, ce sont successivement le chef d'orchestre et critique Fred Goldbeck et le compositeur André Boucourechliev qui y tiennent les chroniques de concert, mais dans deux moments particuliers, des compositeurs sont sollicités : en 1952, à l'occasion du festival "L'Euvre du xx siècle » et, en 1965-1966, dans l'enquête "La musique sérielle aujourd'hui ", menée par Boucourechliev dans les colonnes de Preuves $^{1}$. Comment vont-ils répondre à cette invitation ? Selon quelles modalités vont-ils investir ces espaces médiatiques?

Les PUBLICATIONS DE COMPOSITEURS DANS LA REVUE MUSICALE, N ${ }^{\circ} 212$, AVRIL 1952, NUMÉRO SPÉCIAL « L'EUUVRE DU XX ${ }^{\mathrm{E}}$ SIÈCLE »

La Revue Musicale, fondée en 1920 par Henry Prunières, consacre en avril 1952 un numéro spécial qui anticipe le festival « L'Euvre du Xx siècle » et vise à éclairer le public sur sa programmation proprement musicale. Il sera complété par la publication intégrale des livrets de Wozzeck et Billy Budd dans la livraison de mai. Avec un mois d'avance, il contrepointe le numéro 15 de Preuves qui, sur un ton plus idéologique, livre clairement les objectifs du festival dans sa globalité : «à la campagne de dénigrement conduite par les tenants du pompiérisme, du racisme et du stalinisme, nous avons choisi d'opposer des chefs-d'œuvre de l'art et la pensée libre apparus depuis 50 ans » (Rougemont 1952, p. 1) $)^{2}$.

Ce numéro est coordonné par Nicolas Nabokov qui, tout en livrant des informations sur les contenus des œuvres jouées, y explicite son projet à la fois esthétique et politique. Pour ce faire, il a choisi de s'adresser tant à des compositeurs qu'à des critiques musicaux. Les compositeurs retenus sont (dans l'ordre de la publication) : lui-même, Gian Francesco Malipiero, Virgil Thomson, Zoltán Kodály, Yves Baudrier, Pierre Schaeffer, Antoine Duhamel, Frank Martin, Pierre Boulez (BarthelCalvet 2018, p. 72-74). Certains d'entre eux sont sollicités pour des articles informatifs, pas fondamentalement différents de ceux demandés à des critiques ou musicologues. Dans ce cas, ce sont des compositeurs qui, en marge de leur activité créatrice, exercent aussi des fonctions de journaliste ou de chercheur (par exemple Malipiero, Thomson ou Kodály), et il est, au fond, difficile de savoir à quel titre ils parlent.

L'exemple de Malipiero est tout à fait emblématique de cette ambiguïté et des malentendus qu'elle induit. Son texte "Le renouveau musical italien » s'insère dans le groupe des articles qui dessinent les tendances esthétiques de la création musicale au $\mathrm{XX}^{\mathrm{e}}$ siècle dans différents pays européens et il en partage, il faut le dire, une certaine banalité de ton (voir Malipiero 1952). Mais c'est surtout son refus de se positionner quant à la création contemporaine qui traduit sa difficulté à savoir à quel titre il est

1 Ces deux moments sont contemporains du démarrage, puis de la débâcle du Congrès pour la liberté de la culture.

2 Cette livraison de Preuves ne présente pas, à part Nabokov, d'interventions de compositeurs et comprend : trois articles sur la musique (dont une interview de Casals : " Musique et liberté »), six sur la littérature, deux sur la peinture, ainsi que des réponses à l'enquête sur le réalisme socialiste, qui se poursuit sur plusieurs numéros. 
sollicité. À la fin de son texte, en effet, il ajoute une lettre mentionnant qu'on - très certainement Nabokov - lui a demandé de s'exprimer personnellement sur les développements récents de la musique italienne, mais qu'il s'y refuse, déclarant qu'il n'a " pas voulu juger [ses] confrères " et se limitant à un exposé factuel des récentes œuvres dodécaphoniques de Dallapiccola (voir Malipiero 1952).

Passant sous silence sa propre activité compositionnelle, Nabokov lui-même, dans son texte inaugural, "Introduction à L'Euvre du xx siècle ", prend la parole exclusivement en tant qu'organisateur de la manifestation et observateur de son siècle (Nabokov 1952). D'une certaine manière, il y anticipe le reproche qui lui sera fait d'avoir trop centré sa programmation sur la première moitié du Xx siècle, en justifiant ce choix : pour lui, l'avant-garde de cette époque, par son « esprit d'expérimentation et d'innovation » constitue un modèle pour la création musicale qu'à l'aube des années 1950 il juge encore trop balbutiante. Si rétrospectivement, ce point de vue peut surprendre, force est de constater qu'en 1951-1952, la flamboyante décennie qui va suivre n'en était qu'à ses premières étincelles. Outre cette orientation esthétique, Nabokov affirme la dimension politique de l'engagement de sa démarche qui prône la liberté de la création dans la conclusion de son texte où il renvoie dos à dos art nazi et jdanovisme :

Seul le $\mathrm{xx}^{\mathrm{e}}$ siècle a vu des politiciens s'improvisant professeurs d'harmonie, de composition et d'esthétique, vouant aux gémonies des chefs-d'œuvre, couvrant de grands artistes d'insultes dont la bassesse n'était dépassée que par le ridicule, et imposant une production musicale " mise au pas " des textes serviles, le style le plus plat, et des lignes de conduite " racistes » et des esthétiques "progressistes » manifestement imaginées pour faire désespérer de la race humaine et du progrès artistique (ibid., p. 8).

Deux compositeurs néanmoins exposent leur démarche créatrice qu'ils situent dans la trajectoire de l'évolution musicale : il s'agit de Pierre Schaeffer avec "L'objet musical ", extrait de son ouvrage $A$ la recherche d'une musique concrète, à paraître au Seuil la même année, et de Pierre Boulez avec son fracassant "Éventuellement... » qui ne sera repris qu'une dizaine d'années plus tard dans Relevés d'apprenti, au Seuil également (Boulez 1966). Ces deux textes font écho, respectivement, aux Concerts de musique concrète des 21 et 25 mai et à la création de Structures Ia pour deux pianos par Boulez lui-même et Messiaen, le 7 mai $^{3}$.

Le texte de Schaeffer s'inscrit dans le projet d'une explicitation des enjeux de la création musicale en ce tout début des années 1950 : s'il s'ouvre sur une définition négative de l'objet musical en indiquant tout ce qu'il n'est pas, il donne ensuite des exemples tout à fait compréhensibles pour le grand public et les met en relation avec d'autres œuvres du $\mathrm{Xx}^{\mathrm{e}}$ siècle, en évoquant par exemple, la possibilité de réaliser avec des sons concrets une Klangfarbenmelodie schönbergienne (voir Schaeffer 1952). 
Boulez adopte d'emblée un ton polémique dans ce texte devenu célèbre et dans lequel, avant d'expliciter de manière fort technique la mise en œuvre du sérialisme intégral, il dénonce les postures de ses contemporains - qu'ils soient compositeurs, commentateurs ou simples mélomanes -, qualifiés tour à tour de «sourds", « libertaires ou libertins ", " attardés ", "indulgents qui considèrent la dodécaphonie comme une maladie vénérienne ", avant de lancer sa fameuse diatribe : "Affirmons, à notre tour, que tout musicien qui n'a pas ressenti - nous ne disons pas compris, mais bien ressenti - la nécessité du langage dodécaphonique est INUTILE. Car toute son œuvre se place en deçà des nécessités de son époque » (Boulez 1952, p. 119).

Outre le scandale provoqué par cette formule dans les cercles musicaux, son écho dans l'espace médiatique du Congrès pour la liberté de la culture mérite que l'on s'y arrête. En effet, deux ans plus tard, en juillet 1954, Denis de Rougemont publie dans Preuves un long texte intitulé "Il n'y a pas de "musique moderne" ", précédé d'une introduction de Fred Goldbeck, responsable de la programmation de musique de chambre dans le festival de 1952. Tout en plaidant la nécessité de diffuser plus de musique contemporaine pour habituer le public, de Rougemont y dénonce l'omniprésence étouffante et insidieuse de l'histoire vécue comme " nécessité ", y compris chez les compositeurs qui veulent construire leur démarche contre elle. Tous, selon lui, écrivent de la musique

en connaissance de toute l'histoire de la musique. De là sans doute le ton crispé, voire coléreux de beaucoup de jeunes théoriciens. On les sent bien plus affectés par les résistances qu'ils prévoient que joyeux de leurs découvertes. Ils font ces découvertes contre les autres, qu'ils traitent volontiers d'imbéciles, ou soupçonnent même de mauvaise foi. C'est qu'ils se placent et se regardent dans l'Histoire. Il semble que leur principal souci soit de s'intégrer dans une évolution qu'ils déclarent " nécessaire ", dans on ne sait quelle logique hégélienne de l'Histoire. Ils parlent beaucoup des "nécessités de l'époque " empruntant au vocabulaire économique et politique. Or si l'on peut prouver que l'auto produite par une petite usine est invendable, pour des raisons précises de prix de revient, et ne correspond donc plus aux «nécessités de l'époque » et de nos grands marchés, il n'est nullement prouvé que l'œuvre d'un compositeur non-dodécaphonique « est INUTILE... et sa place en-deçà des nécessités de son époque ". Les " nécessités nouvelles de la musique ", que l'on invoque, ne sont telles que pour l'oreille et l'intelligence d'un très petit groupe d'hommes connaissant toute l'histoire des techniques musicales (Rougemont 1954, p. 76) ${ }^{4}$.

Visant directement le pamphlet boulézien, cette dénonciation d'une omniprésence de l'histoire, à la fois falsificatrice et stérilisante, reviendra par la suite régulièrement dans un certain nombre de chroniques musicales de Preuves, en particulier sous la plume de Fred Goldbeck. Or, paradoxalement, dans ce numéro spécial de La Revue Musicale, c'était bien selon la perspective du « sens de l'histoire » que les compositeurs

4 L'auteur précise en note de bas de page que l'article de Boulez est « une étude par ailleurs importante et fort intelligente». 
étaient invités à interroger la création musicale, ce que leurs textes reflètent de manière diversifiée.

\section{L'ENQUÊTE « LA MUSIQUE SÉRIELLE AUJOURD'HUI » D'ANDRÉ BOUCOURECHLIEV}

L'enquête est une forme d'écrit médiatique qui apparaît assez fréquemment dans Preuves. Par exemple, au moment de "L'Euvre du Xx $x^{\mathrm{e}}$ siècle », le journal avait présenté sur plusieurs numéros une "enquête sur le réalisme socialiste » menée auprès de peintres et d'intellectuels. L'enquête intitulée "La musique sérielle aujourd'hui » et menée par un compositeur, André Boucourechliev, collaborateur à Preuves d'octobre 1962 à avril 1969, se déroule de novembre 1965 (n 177) à mai 1966 (n 183) avec la synthèse de "l'enquêteur », six mois plus tard en octobre 1966 (nº 188).

Le projet est bâti sur une synthèse de sept pages soumise aux compositeurs sollicités (voir Boucourechliev 1965a). Prenant une photographie large des pratiques créatrices qu'il peut observer en 1965, Boucourechliev y décline un certain nombre de questions mises en exergue dans la marge du texte : le public et la relation compositeur-auditeur, les musiques concrète et électronique et les problèmes de perception acousmatique, l'ouverture de l'œuvre, l'aléatoire, le rapport de la musique aux sciences et aux arts, etc., tout ceci dans une vision assez européocentrée. Il est à noter que certaines questions pourtant au centre de débats récents (comme par exemple, la question de la notation, très discutée à Darmstadt en 1964, ou le théâtre musical) ne sont pas abordées. Fait significatif, Boucourechliev emploie toujours l'expression « la musique dite sérielle ", interrogeant ainsi, dans sa formulation même, la légitimité de ce terme. Ce sera d'ailleurs une des questions posées aux personnes interrogées : "que signifie le terme "sériel"?".

Dans cet ensemble assez foisonnant, deux interrogations vont surtout retenir l'attention des contributeurs. Il s'agit des deux premières questions, qui portent sur le champ historiographique :

- La musique sérielle consacre-t-elle une rupture dans la continuité de notre civilisation musicale?

- La musique dite sérielle a voulu intégrer, généraliser, transcender même ses recherches grammaticales rigoureuses dans une poétique aux plus vastes aspects, jusqu'à dissoudre la notion étroite de système dans une notion beaucoup plus large de style. [...] Cette communauté dans la diversité qui en résulte [...] peut-elle être considérée comme l'expression stylistique d'une époque (ibid., p. 20-21)?

Sur cette diversité des questionnements, Boucourechliev a souhaité faire s'exprimer une multiplicité de points de vue. C'est la raison pour laquelle il sollicite non seulement des compositeurs et musiciens (Bruno Maderna, Gilbert Amy, Elliott Carter, Iannis Xenakis, Betsy Jolas, José Vicente Asuar, Pierre Schaeffer, Henri Pousseur, Olivier Messiaen, Mauricio Kagel, Boguslaw Schäffer, Luis de Pablo, Luciano Berio, John Cage, Earle Brown, Kazimierz Sikorski, Maurice Leroux) et des critiques musicaux (Fedele d'Amico, Jacques Lonchampt, Claude Samuel, Claude Rostand, Maurice Fleuret, Stefan Kisielewski), mais aussi des intellectuels (Abraham Moles et François le Lionnais) et des artistes (Jean-Louis Barrault, René Char et André Masson). Les compositeurs - qui représentent la majorité des contributeurs - reflètent la diversité 
des orientations stylistiques de l'époque et Boucourechliev n'a pas hésité à faire

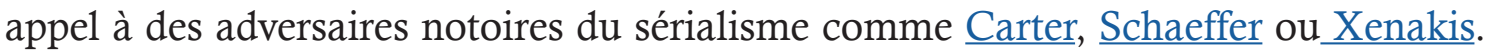
Cependant, certaines figures de l'avant-garde musicale - et non des moindres - n'apparaissent pas : Boulez, Ligeti, Nono, entre autres. Il est vraisemblable qu'ils n'ont pas donné suite à la sollicitation de Boucourechliev dont on imagine difficilement qu'il ne les ait pas contactés.

Si les termes dans lesquels il s'est adressé à chaque contributeur ne sont pas connus, on peut penser qu'il leur a laissé une grande latitude quant à l'orientation des réponses, comme en témoigne la lettre adressée à Iannis Xenakis le 2 juin 1965 :

Cher Yanakis,

Voici le texte d'introduction à la grande enquête sur la " Musique Sérielle Aujourd'hui » que je commence dans la revue PREUVES, et dont je t'avais parlé.

Tu es évidemment directement concerné par cette enquête et ta collaboration est essentielle.

L'enquête veut constituer un ensemble de témoignages constructifs sur la pensée musicale actuelle et ses perspectives d'avenir, et c'est, dans ce sens, au développement le plus libre et le plus personnel de tes idées qu'elle t'invite.

La norme rédactionnelle a été fixée à quatre pages dactylographiées environ, mais tu peux aller jusqu'à six (Boucourechliev 1965b).

La diversité des textes reçus, dans leur longueur comme leur contenu, donne à penser que tous les contributeurs se sont vu accorder la même liberté. Ces textes constituent autant de variations sur les thématiques proposées et instaurent selon des modalités très diversifiées un dialogue plus ou moins explicite entre l'enquêteur et les contributeurs. Certains reprennent la trame des questions, ce qui a tendance à donner un aspect un peu décousu à leur texte ( $\underline{\text { Jolas, }} \underline{\text { de Pablo }}$ ). D'autres reprennent les questions de Boucourechliev dans un texte argumenté qui vise à défendre leur propre pratique compositionnelle, comme Maderna ou encore plus, Schaeffer. D'autres encore ne reprennent que l'esprit général de l'introduction de Boucourechliev, ne répondent pas à ses questions et produisent un texte centré sur une position esthétique ou compositionnelle : Pousseur, alors en pleine élaboration de Votre Faust, ou Berio, qui se tourne aussi à cette époque vers le théâtre instrumental. Il y a enfin ceux comme Cage, Stockhausen ou Xenakis qui font... toute autre chose que de s'inscrire, même de manière éloignée, dans la problématique. Avec respectivement "L'unité du temps musical " (Stockhausen 1966) et "La voie de la recherche et de la question » (Xenakis 1965), Stockhausen et Xenakis profitent de l'espace médiatique qui leur est offert pour exposer dans une revue généraliste leurs réflexions théoriques en cours de développement.

L'éventail des productions va donc du texte d'opinion relatant un parcours personnel à un véritable écrit théorique. Il faut souligner le fait qu'il s'agit parfois de textes assez compliqués et techniques - souvent beaucoup plus que l'introduction de Boucourechliev - et que leurs auteurs semblent avoir oublié, ou délibérément ignoré, qu'ils s'adressent au grand public, fût-il cultivé. La plupart d'entre eux se positionnent par rapport aux deux points historiographiques soulignés plus haut. Sur la question du sérialisme comme rupture dans la " civilisation musicale », une majorité se déclare plutôt, et de manière un peu surprenante, pour l'inscription dans une continuité de 
l'évolution. En revanche, la question du sérialisme comme " expression stylistique d'une époque » donne lieu à des réponses ambiguës, certains comprenant cette formulation comme "mode de pensée " influencé par le sérialisme, d'autres estimant que le terme de sérialisme est devenu fourre-tout et a perdu beaucoup de sa pertinence.

Par la conjugaison des questions destinées à stimuler les réactions des compositeurs sollicités et de la grande liberté qu'il leur accorde, Boucourechliev obtient une extraordinaire mosaïque de points de vue théoriques ou d'opinions qui sont comme le miroir de la diversité des expressions artistiques qu'il relève en introduction. En sollicitant les compositeurs sur l'actualité musicale, cette enquête reprend d'une certaine manière les interrogations soulevées dans le numéro spécial "L'Euvre du xx siècle » de La Revue Musicale - peut-être parce que de telles thématiques s'inscrivent dans la ligne éditoriale des publications pilotées par le Congrès pour la liberté de la culture et répondent aux attentes de leur lectorat. La démarche de Boucourechliev s'inscrit néanmoins dans une tout autre ligne que celle défendue par Fred Goldbeck et Denis de Rougemont reprochant aux jeunes compositeurs d'avant-garde leur obsession de l'histoire. Certains préféreront cependant répondre " hors sujet » et, à l'instar d'un Stockhausen ou d'un Xenakis, livrer un texte projetant vers l'avenir leur propre pratique créatrice.

Dans les deux cas, les compositeurs se voient ouvrir un espace médiatique plus large que celui des publications destinées à un lectorat de musiciens ou mélomanes dans lesquelles ils ont 1'habitude de s'exprimer. Confrontés à ce changement, ils réagissent et apprécient de manière variable la façon dont ils peuvent s'adresser au grand public, et c'est ce qui produit la très grande diversité de ton et de contenu de ces textes. De cette diversité et du format de « collection » inhérent à ces deux projets, l'un de panorama, l'autre d'enquête, émerge une forme de débat qui correspond bien à l'esprit des publications du Congrès.

\section{BIBLIOGRAPHIE}

Barthel-Calvet, Anne-Sylvie (2018), « Targeting New Music in Postwar Europe. American Cultural Diplomacy in the Crafting of Art Music Avant-Garde Scenes », dans Frédéric Ramel, Cécile PrévostThomas (dir.), International Relations, Music and Diplomacy. Sounds and Voices on the International Stage, Basingstoke, Palgrave Macmillan, p. 65-90.

Boucourechliev, André (1965a), «La musique sérielle aujourd'hui », Preuves, n 177 (novembre), p. 2027, https://dicteco.huma-num.fr/fr/article/40337, consulté le 7 août 2019.

Boucourechliev, André (1965b), Lettre à Iannis Xenakis (2 juin), Archives Famille Xenakis, dossier Écrits 4/11.

Boulez, Pierre (1952), "Éventuellement... ", La Revue Musicale, no 212 (avril), p. 119, https://dicteco.huma-num.fr/fr/article/40322, consulté le 7 août 2019.

Boulez, Pierre (1966), "Éventuellement... », Relevés d'apprenti, Paris, Seuil, p. 183-204, https://dicteco.huma-num.fr/fr/anthology/39574, consulté le 7 août 2019.

Carroll, Mark (2003), Music and Ideology in Cold War Europe, Cambridge, Cambridge University Press.

Giroud, Vincent (2015), Nicolas Nabokov. A Life in Freedom and Music, Oxford, Oxford University Press. 
Grémion, Pierre (1995), Intelligence de l'anticommunisme. Le Congrès pour la liberté de la culture à Paris 19501975, Paris, Fayard.

Malipiero, Gian F. (1952), « Le renouveau musical italien », La Revue Musicale, n 212 (avril), p. 29, https://dicteco.huma-num.fr/fr/article/40310, consulté le 7 août 2019.

Nabokov, Nicolas (1952), "Introduction à L'Euvre du xx siècle », La Revue Musicale, no 212 (avril), p. 5-8, https://dicteco.huma-num.fr/fr/article/40319, consulté le 7 août 2019.

Preuves, $\mathrm{n}^{\circ} 177$, novembre 1965 , p. 20-38; no 178, décembre 1965 , p. 27-42; no 179, janvier 1966, p. 39$55 ; \mathrm{n}^{\circ} 180$, février 1966 , p. $30-46 ; \mathrm{n}^{\circ} 181$, mars 1966 , p. $37-47 ; \mathrm{n}^{\circ} 183$, mai 1966 , p. $51-57 ;$ n $^{\circ} 188$, octobre 1966, p. 38-44.

La Revue Musicale (1952), n 212 (avril), numéro spécial « L'Euvre du xx siècle ».

Rougemont, Denis de (1952), " "L'Euvre du xx siècle", une réponse ou une question ? ", Preuves, $\mathrm{n}^{\circ} 15$ (mai), p. 1.

Rougemont, Denis de (1954), "Il n'y a pas de "musique moderne" ", Preuves, no 41 (juillet), p. 75 77.

Schaeffer, Pierre (1952), "L'objet musical », La Revue Musicale, $\mathrm{n}^{\circ} 212$ (avril), p. 65-76, https://dicteco.huma-num.fr/fr/article/40955, consulté le 7 août 2019.

Stockhausen, Karlheinz (1966), "L'unité du temps musical ", Preuves, n 180 (février), p. 31-35, https://dicteco.huma-num.fr/fr/article/41029, consulté le 7 août 2019.

Xenakis, Iannis (1965), « La voie de la recherche et de la question (Formalisation et axiomatisation de la musique », Preuves, no 177 (novembre), p. 33-36, https://dicteco.huma-num.fr/fr/article/40985, consulté le 7 août 2019. 